\title{
How Long Do We Need to Restrict Weight Bearing after Fixation of Pediatric Femur Fractures?
}

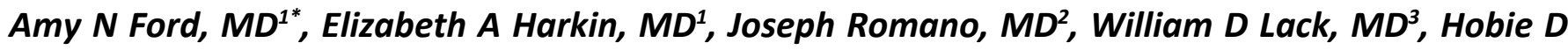 \\ Summers, $M D^{1}$ and Joseph $B$ Cohen, $M D^{1}$
}

${ }^{1}$ Loyola University Medical Center, USA

${ }^{2}$ Vanderbilt University Medical Center, USA

${ }^{3}$ University of Washington Medical Center, USA

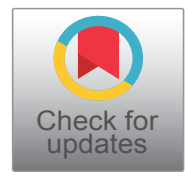

*Corresponding author: Amy Ford, MD, Loyola University Medical Center, 2160 South $1^{\text {st }}$ Avenue, Building 105, Suite 1700, Maywood, IL 60153, USA, Tel: (623)-826-2559, Fax: (708)-216-5858

\begin{abstract}
Background: Surgical management of pediatric femur fractures is accomplished with intramedullary nailing or plate fixation. These fractures heal quickly with a low incidence of complications, although there is a paucity of evidence on how long postoperative weight bearing restrictions need to be in place or whether they are even necessary at all. The objectives of this study are to compare methods of fixation of pediatric femur fractures and postoperative weight bearing protocols and to correlate these with healing time and complication rates.

Methods: This is a retrospective cohort study performed at a Level 1 Trauma Center, investigating 60 patients ages 4 to 15 with 63 femoral shaft fractures fixed with intramedullary nailing or plate osteosynthesis. Patients underwent closed, percutaneous, or open reduction of their fracture, followed by locked or flexible intramedullary nailing or plate fixation. Postoperative weight bearing protocols were at the discretion of the treating surgeons. Main outcome measurements included time to callus formation, complications, and time to weight bearing.

Results: 63 fractures were treated using rigid locked intramedullary nails (10), flexible unlocked intramedullary nails (14), and plate fixation (39). No significant difference in time to callus formation or complication rate was found between the types of fixation. There was a significant association between locked nailing and the allowance of weight bearing postoperatively ( $p<0.01)$, but otherwise no association was found between the methods of reduction or fixation and the weight bearing protocol. All patients in the study developed callus over multiple cortices by 6 weeks and ultimately reached radiographic union.
\end{abstract}

Conclusion: Though there was variability in postoperative treatment of pediatric femur fractures, this study demonstrates no statistically significant difference between methods of reduction, fixation, or postoperative weight bearing protocols in radiographic outcomes. Further investigation may be needed to ensure adequate power to support definitive conclusions.

\section{Keywords}

Pediatric, Femur, Fixation, Weight bearing, Callus

\section{Introduction}

Within the past 2 decades, there has been an overall trend toward increased surgical management of pediatric femur fractures. This is particularly true in the 5 to 10-year-old age group, in which the use of operative treatment has more than doubled [1]. The 3 commonly used modes of fixation for femur fractures in children and adolescents are flexible intramedullary nailing, locked intramedullary nailing, and submuscular plating. Multiple studies have validated the use of plate osteosynthesis and intramedullary nailing in the fixation of these fractures, with each technique having its own unique set of indications and considerations [2,3].

When utilized in the appropriate patients, these operative methods have shown successful outcomes with an overall low risk of complications [4-6]. Healing of pediatric femur fractures is reliable, with a reported average

Citation: Ford AN, Harkin EA, Romano J, Lack WD, Summers HD, et al. (2020) How Long Do We Need to Restrict Weight Bearing after Fixation of Pediatric Femur Fractures?. Int Arch Orthop Surg 3:015. doi.org/10.23937/2643-4016/1710015

Accepted: January 04, 2020; Published: January 06, 2020

Copyright: (C) 2020 Ford AN, et al. This is an open-access article distributed under the terms of the Creative Commons Attribution License, which permits unrestricted use, distribution, and reproduction in any medium, provided the original author and source are credited. 
time to radiographic union of approximately 6 weeks for compression plating, 6 weeks for percutaneous submuscular plating, and 8 weeks for flexible nailing $[7,8]$.

Though several studies demonstrate consistency in healing time and clinical outcomes between these different fixation methods, postoperative weight bearing protocols vary widely between providers, with some requiring non-weight bearing until evidence of radiographic healing, others allowing touchdown weight bearing for 6 weeks with progressive advancement thereafter, and some even further immobilizing in spica casts [4-12]. Periods of weight bearing protected by assistive device also vary depending on surgeon preferences [13]. The time to full weight bearing after fracture fixation has been investigated as an outcome measure in comparison studies of surgical technique, but such research may be confounded by the instructions of the treating surgeon.

The purpose of this study is to evaluate differences in fixation and postoperative weight bearing protocols for pediatric femur fractures at our institution. We attempted to identify any association between the method of reduction and fixation, the postoperative weight bearing instructions, the time to radiographic callus formation, and the incidence of postoperative complications.

\section{Methods}

After obtaining Institutional Review Board (IRB) approval, our institutional database was queried from 2007 to 2017 to identify pediatric patients between the ages of 4 and 15 who underwent surgical treatment of a femur fracture (CPT codes 27244, 27245, $27506,27507,27511)$. Femoral head and neck fractures and intra-articular distal femur fractures were excluded from the initial search. Retrospective chart review was performed using the electronic medical record (EPIC Hyperspace, EPIC Systems Inc., Verona, WI) to further exclude any physeal fractures, pathologic fractures, open fractures, and ipsilateral injuries that precluded postoperative weight bearing on the injured limb. Patients with neurovascular injury and those who were nonambulatory at baseline were also excluded. The final cohort included fractures of $A O /$ OTA classification 31A, 32A, 32B, 32C, and 33A.

Patient demographics (Table 1 ) and clinical data were recorded, including age, gender, height, weight, laterality of injury, mechanism of injury, and concomitant injuries. Radiographic data included fracture pattern and location, as well as the timing to presence of callus over multiple cortices on postoperative radiographs. Operative data included fracture reduction technique (direct/percutaneous/indirect), type of implant used, and presence or absence of compression through the implant. Postoperative data included complications and weight bearing in-

\section{structions.}

Our study population included 60 patients with 63 fractures after excluding patients for concomitant injuries limiting postoperative weight bearing (14), baseline nonambulatory status (6), open fracture (6), physeal or articular involvement (4), pathologic fracture (2), and follow-up less than 1 month (2). There were 8 surgeons involved in the care of these patients with subspecialty training in trauma, oncology, and pediatrics. Implant choice was based on fracture pattern, patient characteristics, and surgeon preference. Fixation was performed by locked intramedullary nailing, flexible intramedullary nailing, or submuscular plating. Flexible nailing was preferred by our pediatric orthopaedic surgeon, while submuscular plating was performed primarily by our orthopaedic trauma surgeons. Flexible nailing was only performed in patients under 50 kilograms with length-stable fracture patterns (32A2 or $32 \mathrm{~A} 3$ ). Standard surgical techniques were used throughout.

Table 1: Demographics.

\begin{tabular}{|l|l|}
\hline Total Number of Patients & 60 \\
\hline Male & 45 \\
\hline Female & 15 \\
\hline Age (average) & 9.52 years \\
\hline Height (average) & $142.11 \mathrm{~cm}$ \\
\hline Weight (average) & $38.62 \mathrm{~kg}$ \\
\hline BMI (average) & $20.70 \mathrm{~kg} / \mathrm{m}^{2}$ \\
\hline AO Classification & \\
\hline 31-A3 & 1 \\
\hline 32-A1 & 9 \\
\hline 32-A2 & 14 \\
\hline 32-A3 & 33 \\
\hline 32-B1 & 1 \\
\hline 32-B2 & 1 \\
\hline 32-B3 & 1 \\
\hline 32-C1 & 1 \\
\hline 33-A1 & 2 \\
\hline Mechanism & 14 \\
\hline MVC & 16 \\
\hline Pedestrian struck by auto & 16 \\
\hline Bicyclist struck by auto & 3 \\
\hline Fall & 15 \\
\hline Crush & 6 \\
\hline Contact sport & 5 \\
\hline Other collision & 2 \\
\hline Implant & \\
\hline Locked IMN & 10 \\
\hline Flexible IMN & \\
\hline Plate & \\
\hline
\end{tabular}

BMI: Body mass index; MVC: Motor vehicle collision; IMN: Intramedullary nail. 
Postoperatively, all patients were followed through radiographic union, with a mean follow-up duration of 18.31 months (range 3-104 months). Although they were followed at varying intervals of time, all patients had an early postoperative follow-up at approximately 2 weeks and another postoperative follow-up at approximately 6 weeks. Postoperative radiographs were not routinely obtained at 2 weeks but were consistently obtained at 6 weeks. Postoperative physical therapy was ordered according to surgeon protocol, and this varied by surgeon. All radiographs were analyzed by 1 author for the presence of callus over multiple bony cortices on orthogonal views, which was used as an indication of successful healing.

Clinical outcomes included the allowance of postoperative weight bearing, the presence of callus over multiple cortices, ultimate radiographic union, and postoperative complications.

The Kruskal-Wallis test was used to determine whether any statistically significant associations were present among the weight bearing groups when data was non-normally distributed, such as methods of reduction and fixation. Fisher's exact test was used to analyze complication rate. Post hoc tests were then further employed to specify the significant associations.

\section{Results}

The study group was comprised of 63 fractures in 60 patients. Locked intramedullary nailing was performed for 10 fractures (15.9\%), flexible intramedullary nailing for 14 fractures (22.2\%), and submuscular plating for 39 fractures $(61.9 \%)$. Open reductions were performed in 19 cases (30.2\%), percutaneous reductions in 8 cases $(12.7 \%)$, and indirect reductions in the remaining 36 cases (57.1\%). 18 fractures (28.6\%) were compressed, either through the use of a screw placed eccentrically in the hole of the plate, or through the placement of a lag screw followed by neutralization plating. 43 patients (68.3\%) were made non-weight bearing immediately postoperatively, though $8(12.7 \%)$ of these were presumably to allow for soft tissue rest and were advanced in their weight bearing status shortly afterwards. At 2 weeks postoperatively, 8 patients $(12.7 \%)$ were allowed to be touchdown weight bearing, and 20 patients $(31.7 \%)$ were allowed to be full weight bearing.

Surgeons were most restrictive with postoperative weight bearing when fixation was performed with flexible intramedullary nails, and weight bearing was most readily advanced when fixation was performed with locked intramedullary nails. By 2 weeks postoperatively, all patients who underwent locked intramedullary nailing were allowed to be full weight bearing on the affected extremity. On the other hand, 11/14 (78.6\%) of those stabilized with flexible nails were still required to continue non-weight bearing on the extremity. Postoperative protocols for submuscular plating were more variable. By 2 weeks postoperatively, 24/39 (61.5\%) of the patients who underwent submuscular plating were non-weight bearing, while $7 / 39(17.9 \%)$ were touchdown weight bearing and $8 / 39(20.5 \%)$ were full weight bearing on the extremity. A small number of patients in each subset were restricted in weight bearing between immediately and 2 weeks postoperatively; though the reason for this was not explicitly stated, it was presumed to be for soft tissue rest since this practice was independent of radiographic findings. This occurred most frequently in those who underwent submuscular plating. By 6 weeks postoperatively, all but 3 patients in the study were allowed to be full weight bearing, with the final 3 kept touchdown weight bearing until their subsequent follow-up appointment. There was a statistically significant difference in the amount of weight bearing allowed at 2 weeks between locked nailing and the other two groups $(p<0.01)$; however, when flexible nailing and plating were compared, there was no significant difference $(p=0.37)$ (Table 2 and Table 3$)$.

Patient weight did not appear to be a factor in the

Table 2: Weight Bearing at 2 Weeks Postoperatively.

\begin{tabular}{|c|c|c|c|c|c|}
\hline \multicolumn{2}{|l|}{ Intervention } & \multirow[t]{2}{*}{ NWB } & \multirow[t]{2}{*}{ TDWB } & \multirow[t]{2}{*}{ WBAT } & \multirow{2}{*}{$\begin{array}{l}\text { p-value } \\
<0.01\end{array}$} \\
\hline Implant & & & & & \\
\hline & Locked IMN ( $n=10)$ & $0(0 \%)$ & $0(0 \%)$ & $10(100 \%)$ & \\
\hline & Flexible IMN $(n=14)$ & $11(78.6 \%)$ & $1(7.1 \%)$ & $2(14.3 \%)$ & \\
\hline & Plate $(n=39)$ & $24(61.5 \%)$ & $7(17.9 \%)$ & $8(20.5 \%)$ & \\
\hline \multirow[t]{4}{*}{ Reduction } & & & & & 0.11 \\
\hline & Direct $(n=19)$ & $11(57.9 \%)$ & $4(21.1 \%)$ & $4(21.1 \%)$ & \\
\hline & Percutaneous $(n=9)$ & $8(88.9 \%)$ & $0(0 \%)$ & $1(11.1 \%)$ & \\
\hline & Indirect $(n=35)$ & $16(45.7 \%)$ & $4(11.4 \%)$ & $15(42.9 \%)$ & \\
\hline \multirow[t]{3}{*}{ Compression } & & & & & 0.95 \\
\hline & Compressed $(n=18)$ & $10(55.6 \%)$ & $2(11.1 \%)$ & $6(33.3 \%)$ & \\
\hline & Not Compressed $(n=45)$ & $25(55.6 \%)$ & $6(13.3 \%)$ & $14(31.1 \%)$ & \\
\hline
\end{tabular}

NWB: Non-weight bearing; TDWB: Touchdown weight bearing; WBAT: Weight bearing as tolerated; IMN: Intramedullary nail. 
Table 3: Kruskal-Wallis Test for Weight Bearing.

\begin{tabular}{|c|c|c|}
\hline \multicolumn{3}{|l|}{ Weight Bearing by Implant } \\
\hline H-statistic: & 4.62289 & \multirow{2}{*}{$\begin{array}{l}\text { Degrees of } \\
\text { freedom: } 2\end{array}$} \\
\hline p-value: & 0.000023 & \\
\hline \multicolumn{3}{|c|}{ Dunn $p$-values, further adjusted by the Benjamini-Hochberg FDR method (significant $p<0.017$ ) } \\
\hline Locked vs. Flexible IMN & \multicolumn{2}{|l|}{$p=0.000039$} \\
\hline Locked IMN vs. Plate & \multicolumn{2}{|l|}{$p=0.000039$} \\
\hline Flexible IMN vs. Plate & \multicolumn{2}{|l|}{$p=0.373169$} \\
\hline \multicolumn{3}{|l|}{ Weight Bearing by Reduction } \\
\hline H-statistic: & 4.3387 & \multirow{2}{*}{$\begin{array}{l}\text { Degrees of } \\
\text { freedom: } 2\end{array}$} \\
\hline p-value: & 0.11425 & \\
\hline \multicolumn{3}{|l|}{ Weight Bearing by Compression } \\
\hline H-statistic: & 0.0037 & \multirow{2}{*}{$\begin{array}{l}\text { Degrees o } \\
\text { freedom: } 2\end{array}$} \\
\hline p-value: & 0.95147 & \\
\hline
\end{tabular}

Table 4: Reduction Method by Implant.

\begin{tabular}{|l|l|l|l|l|l|}
\hline & Direct & Percutaneous & Indirect & p-value \\
\hline Implant & & & & $<0.01$ \\
\hline & Locked IMN $(\mathrm{n}=10)$ & $0(0.0 \%)$ & $0(0.0 \%)$ & $10(100.0 \%)$ \\
\hline & Flexible IMN $(\mathrm{n}=14)$ & $1(7.1 \%)$ & $3(21.4 \%)$ & $10(71.4 \%)$ \\
\hline & Plate $(\mathrm{n}=39)$ & $18(46.2 \%)$ & $5(12.8 \%)$ & $16(41.0 \%)$ \\
\hline
\end{tabular}

Table 5: Kruskal-Wallis Test for Reduction Method.

Reduction Method by Implant

\begin{tabular}{|l|l|l}
\hline H-statistic & 3.71632 & Degrees of \\
\hline$p$-value & 0.001002 & freedom: 2
\end{tabular}

Dunn $p$-values, further adjusted by the Benjamini-Hochberg FDR method (significant $p<0.017$ )

\begin{tabular}{|l|l|}
\hline Locked vs. Flexible IMN & $p=0.280686$ \\
\hline Locked IMN vs. Plate & $p=0.002531$ \\
\hline Flexible IMN vs. Plate & $p=0.027118$
\end{tabular}

decision to prevent early weight bearing. The average weight of the non-weight bearing group at 2 weeks was 33.15 kilograms; the touchdown weight bearing group was 48.17 kilograms, and the full weight bearing group was 45.93 kilograms. When locked intramedullary nails were removed from the data set, as these patients tended to be heavier and were uniformly allowed to bear full weight postoperatively, the average weight for the full weight bearing group at 2 weeks was 30.43 kilograms.

Open reductions were performed based on either preference or necessity, with attempted closed reductions at times converted to percutaneous or open in order to obtain an adequate reduction. 19 (30.2\%) fractures were reduced directly, using an open approach to the fracture. The vast majority of these $(94.7 \%)$ utilized plate fixation for fracture stabilization. $8(12.7 \%)$ fractures were reduced percutaneously with an instrument within the fracture site but only visualized on fluoroscopy. The remaining 36
(57.1\%) fractures were reduced indirectly. Fractures treated with locked intramedullary nails were significantly more likely than those treated with plates to be reduced indirectly ( $p<0.01$ ) (Table 4 and Table 5). By 2 weeks postoperatively, surgeons allowed $42.2 \%$ of those who underwent direct reduction and $45.5 \%$ of those who underwent indirect or percutaneous reduction to bear some weight on their fracture. There was no statistically significant correlation between method of reduction and postoperative weight bearing status ( $p=0.11)$ (Table 2 and Table 3$)$.

All patients whose fractures were compressed underwent fixation with plate osteosynthesis. By 2 weeks postoperatively, $33.3 \%$ of these patients were allowed to be full weight bearing, with another $11.1 \%$ being touchdown weight bearing. There was no statistically significant correlation between use of compression and postoperative weight bearing status ( $p=0.95$ ) (Table 2 and Table 3).

There were no cases of nonunion. Radiographs 
were not regularly obtained at the 2 week follow-up visit but were generally obtained at 6 weeks postoperatively. All patients who had radiographs at the 6 week time point demonstrated the presence of callus over multiple cortices. In the 3 patients who missed their 6 week follow-up, all had abundant callus over multiple cortices when they were seen again at 8.5 to 9.5 weeks postoperatively.

There were only 3 notable complications within our study group; 2 of these were from the flexible nail group (13.33\%) and the third was from the plate group $(2.56 \%)(p=0.18)$.

One complication in the flexible nail group was in a 12-year-old male with a short oblique femoral shaft fracture, who was allowed to be touchdown weight bearing at 4.5 weeks and advance as tolerated. He was lost to follow-up until 15.5 weeks postoperatively, when he presented with a re-fracture through his callus after he was tackled while playing football. He underwent plating of his fracture and had uncomplicated healing by 6 weeks.

Another flexible nail complication occurred in an 8 -year-old female who was found to have loss of reduction on radiographs obtained at her 1.5 week follow-up. She underwent revision closed reduction with adjustment of the nails, was kept non-weight bearing until 4 weeks postoperatively, and subsequently healed uneventfully.

The final complication occurred in an 8-year-old male with a transverse midshaft fracture who underwent open reduction and submuscular plating. He was made non-weight bearing but was lost to follow-up until 9 weeks postoperatively and had reportedly been bearing weight on his leg for 1 week by this time. He was at a playground when he twisted and fractured his femur at the level of the most proximal screw. He underwent revision plate fixation with compression through a lag screw and was kept non-weight bearing until 6.5 weeks postoperatively. Radiographs were obtained at that time showing callus over multiple cortices over both of his fractures, and he was allowed to progress weight bearing.

\section{Discussion}

The treatment of choice for pediatric femur fractures remains dictated by the child's characteristics, the fracture pattern, and the treating surgeon's preferences. While locked compression plate fixation has shown at least twice the stiffness and increased torsional stability when compared with titanium elastic nails, flexible nailing has the advantages of minimal soft tissue dissection, faster operative times, and decreased surgical costs, with equivalent outcomes when used in the appropriate patients [14-16]. Plating is accompanied by the potential risks of delayed healing from soft tissue stripping, femoral over- growth, implant failure, and fracture through screw holes $[9,12,17-19]$. Flexible nailing, on the other hand, is theoretically limited to length-stable fractures in smaller patients, with greater risks of loss of reduction, malunion, and a longer time to full weight bearing as a result of decreased stability $[12,20,21]$.

Despite the risks and benefits involved with each method, all have been employed successfully in the treatment of pediatric femur fractures $[13,17,22]$. This patient demographic and injury pattern is typically associated with dependable radiographic healing over a period of 6-8 weeks $[7,8]$. Time to full weight bearing has been used as another outcome measure, reported at an average of 8 weeks for compression plating, 7 weeks for submuscular plating, and 12 weeks for flexible nailing $[4,12]$. However, postoperative weight bearing protocols vary widely between providers and institutions, with some allowing immediate weight bearing with assistive device, others requiring touchdown weight bearing until callus formation, with progressive advancement, and others still preferring non-weight bearing until callus formation [5,7-11,15].

Our study is the first to focus on the variability that exists between different surgeons' postoperative protocols after fixation of pediatric femur fractures, even when stratifying by implant choice. We found that some surgeons required 2 weeks of restricted weight bearing for soft tissue rest, while others chose to forego the resting period entirely. Though immediate weight bearing on locked intramedullary nails was universal, there was greater variability among flexible intramedullary nail and submuscular plate fixation. In those cases, there was no significant correlation between implant used and weight bearing protocol. There was no trend towards increasing patient weight and increasing weight bearing restrictions. We found no association between the use of direct or indirect reduction with the chosen weight bearing protocol, despite the theoretical concern of devitalizing the fracture site through soft tissue stripping. Similarly, we found that the use of compression through the fracture site was not associated with the allowance of earlier weight bearing. There was also no association between method of fixation and radiographic healing, which was relatively robust for all cases.

The plate fixation group was found to have the most variability in postoperative weight bearing protocol. Of particular interest was the cohort of patients who underwent plate fixation and were allowed to bear weight immediately on their fractured extremity. By 2 weeks postoperatively, a total of 15 patients in this cohort were allowed to bear some degree of weight. As above, all of these patients healed uneventfully, and there were no complications in this subset of patients. This suggests that stable plate fixation of femur fractures in 
children may diminish the need for restricted weight bearing, similar to the protocol employed for locked intramedullary nailing.

Limitations of this study include its retrospective nature. This limited data to information available in the medical record, which did not allow for the inclusion of functional outcomes, as these were not adequately or consistently documented. It also limited data collection to those protocols which were arbitrarily decided upon by the surgeon, which left us unable to analyze a greater variety of treatment arms. Additionally, it is difficult to know definitively when patients began full weight bearing, regardless of when they were instructed to begin by their surgeon. Since we were looking specifically at weight bearing protocols, we had to exclude a substantial number of patients who had other factors affecting their postoperative weight bearing. We were thus left with a relatively small sample size, which may have affected the statistical significance of our results and predisposed our findings to sampling bias. Another limitation is the inability to truly gauge the time to callus formation because radiographic data is limited to follow-up appointments during which radiographs are taken. Surgeon and patient variability in scheduling and attending postoperative follow-up visits contributed to missing data. Finally, adequate visualization of all bony cortices on postoperative radiographs was limited by interference from implants, specifically the plates. This has been previously reported as a factor complicating radiographic assessment of fracture healing [23].

In conclusion, children are an inherently irresponsible patient demographic with regards to adherence to postoperative instructions. However, they have also shown themselves to reliably heal their fractures under a variety of treatments and postoperative weight bearing protocols. As a whole, surgeons have not agreed upon the necessary weight bearing restrictions for pediatric femur fractures, particularly those treated with plate fixation. In this study, we found that such practices do not seem to be directly influenced by the use of an open or closed reduction or compression at the fracture site. While fracture pattern may have directed implant choice, we did not find any indication that the injury pattern or patient demographics affected decisions to allow or restrict weight bearing. Our study provides preliminary support that non-weight bearing precautions may not be necessary following plate fixation of pediatric femur fractures because all of our patients healed quickly and robustly. What may be of greater importance is preventing early return to high-level exercises, as 2 of our 3 complications were related to playground and athletic activities. Further studies are needed to investigate the feasibility of allowing children to begin early weight bearing on their fractures after fixation.

\section{Conflict of Interest Statement}

The authors have no conflicts of interest to disclose. No external funding was necessary for the completion of this study.

\section{References}

1. Naranje SM, Stewart MG, Kelly DM, Jones TL, Spence DD, et al. (2016) Changes in the treatment of pediatric femoral fractures: 15-year trends from United States Kids' Inpatient Database (KID) 1997 to 2012. J Pediatr Orthop 36: e81-e85.

2. Ramseier LE, Janicki JA, Weir S, Narayanan UG (2010) Femoral fractures in adolescents: A comparison of four methods of fixation. J Bone Joint Surg Am 92: 1122-1129.

3. Subasi M, Kapukaya A (2006) Treatment options in pediatric femoral shaft fractures. Journal of Orthopaedic Trauma 20: $297-298$

4. Kregor PJ, Song KM, Routt ML, Sangeorzan BJ, Liddell RM, et al. (1993) Plate fixation of femoral shaft fractures in multiply injured children. J Bone Joint Surg Am 75: 17741780.

5. Caird MS, Mueller KA, Puryear A, Farley FA (2003) Compression plating of pediatric femoral shaft fractures. J Pediatr Orthop 23: 448-452.

6. Eren OT, Kucukkaya M, Kockesen C, Kabukcuoglu Y, Kuzgun $U$ (2003) Open reduction and plate fixation of femoral shaft fractures in children aged 4 to 10 . J Pediatr Orthop 23: 190-193.

7. Sink EL, Hedequist D, Morgan SJ, Hresko T (2006) Results and technique of unstable pediatric femoral fractures treated with submuscular bridge plating. J Pediatr Orthop 26: $177-181$

8. Fyodorov I, Sturm PF, Robertson WW Jr (1999) Compression-plate fixation of femoral shaft fractures in children aged 8 to 12 years. J Pediatr Orthop 19: 578-581.

9. Stoneback JW, Carry PM, Flynn K, Pan Z, Sink EL, et al. (2018) Clinical and radiographic outcomes after Submuscular Plating (SMP) of pediatric femoral shaft fractures. J Pediatr Orthop 1-6.

10. Abdelgawad AA, Sieg RN, Laughlin MD, Shunia J, Kanlic EM (2013) Submuscular bridge plating for complex pediatric femur fractures is reliable. Clin Orthop Relat Res 471: $2797-2807$

11. Samora WP, Guerriero M, Willis L, Klingele KE (2013) Submuscular bridge plating for length-unstable, pediatric femur fractures. J Pediatr Orthop 33: 797-802.

12. Sutphen SA, Beebe AC, Klingele KE (2016) Bridge plating length-unstable pediatric femoral shaft fractures. J Pediatr Orthop 36: S29-S34.

13. Ağuş H, Kalenderer O, Eryanilmaz G, Omeroğlu H (2003) Biological internal fixation of comminuted femur shaft fractures by bridge plating in children. J Pediatr Orthop 23: 184189.

14. Porter SE, Booker GR, Parsell DE, Weber MD, Russell GV, et al. (2012) Biomechanical analysis comparing titanium elastic nails with locked plating in two simulated pediatric femur fracture models. J Pediatr Orthop 32: 587-593.

15. Allen JD, Murr K, Albitar F, Jacobs C, Moghadamian ES, et al. (2018) Titanium elastic nailing has superior value to plate fixation of midshaft femur fractures in children 5 to 11 years. J Pediatr Orthop 38: e111-e117. 
16. Sutphen SA, Mendoza JD, Mundy AC, Yang JG, Beebe AC, et al. (2016) Pediatric diaphyseal femur fractures: Submuscular plating compared with intramedullary nailing. Orthopedics 39: 353-358.

17. Kanlic EM, Anglen JO, Smith DG, Morgan SJ, Pesántez RF (2004) Advantages of submuscular bridge plating for complex pediatric femur fractures. Clin Orthop Relat Res 426: 244-251.

18. Caglar O, Aksoy MC, Yazici M, Surat A (2006) Comparison of compression plate and flexible intramedullary nail fixation in pediatric femoral shaft fractures. J Pediatr Orthop B 15: $210-214$

19. Becker T, Weigl D, Mercado E, Katz K, Bar-On E (2012) Fractures and refractures after femoral locking compression plate fixation in children and adolescents. J Pediatr Orthop 32: e40-e46.
20. Moroz LA, Launay F, Kocher MS, Newton PO, Frick SL, et al. (2006) Titanium elastic nailing of fractures of the femur in children. Predictors of complications and poor outcome. J Bone Joint Surg Br 88: 1361-1366.

21. May C, Yen YM, Nasreddine AY, Hedequist D, Hresko MT, et al. (2013) Complications of plate fixation of femoral shaft fractures in children and adolescents. J Child Orthop 7: 235-243.

22. Hedequist DJ, Sink E (2005) Technical aspects of bridge plating for pediatric femur fractures. J Orthop Trauma 19: 276-279.

23. Strotman PK, Karunakar MA, Seymour R, Lack WD (2017) Any cortical bridging predicts healing of supracondylar femur fractures after treatment with locked plating. J Orthop Trauma 31: 538-544. 